\title{
SOME CUBIC MODULAR IDENTITIES OF RAMANUJAN
}

\author{
J. M. BORWEIN, P. B. BORWEIN AND F. G. GARVAN
}

ABstract. There is a beautiful cubic analogue of Jacobi's fundamental theta function identity: $\theta_{3}^{4}=\theta_{4}^{4}+\theta_{2}^{4}$. It is

$$
\begin{aligned}
\left(\sum_{n, m=-\infty}^{\infty} q^{n^{2}+n m+m^{2}}\right)^{3}= & \left(\sum_{n, m=-\infty}^{\infty} \omega^{n-m} q^{n^{2}+n m+m^{2}}\right)^{3} \\
& +\left(\sum_{n, m=-\infty}^{\infty} q^{\left(n+\frac{1}{3}\right)^{2}+\left(n+\frac{1}{3}\right)\left(m+\frac{1}{3}\right)+\left(m+\frac{1}{3}\right)^{2}}\right)^{3} .
\end{aligned}
$$

Here $\omega=\exp (2 \pi i / 3)$. In this note we provide an elementary proof of this identity and of a related identity due to Ramanujan. We also indicate how to discover and prove such identities symbolically.

\section{INTRODUCTION}

In [5] the behaviour of the hypergeometric function ${ }_{2} F_{1}\left(\frac{1}{3}, \frac{2}{3} ; 1 ; \cdot\right)$ was studied and exploited. Central to that paper was the cubic identity, implicit in [6]:

$$
\begin{aligned}
\left(\sum q^{n^{2}+n m+m^{2}}\right)^{3}= & \left(\sum \omega^{n-m} q^{n^{2}+n m+m^{2}}\right)^{3} \\
& +\left(\sum q^{\left(n+\frac{1}{3}\right)^{2}+\left(n+\frac{1}{3}\right)\left(m+\frac{1}{3}\right)+\left(m+\frac{1}{3}\right)^{2}}\right)^{3}
\end{aligned}
$$

where $\omega:=e^{2 \pi i / 3}$, and all sums range over $\mathbb{Z}^{2}$. Identity (1.1) was established in [5] by modular function techniques. It is our purpose here to establish (1.1) by elementary means. For this purpose it is convenient to recall the definition of the Dedekind eta function

$$
\eta(q):=q^{\frac{1}{24}} \prod_{n=1}^{\infty}\left(1-q^{n}\right),
$$

Received by the editors April 2, 1991.

1980 Mathematics Subject Classification (1985 Revision). Primary 33D10; Secondary 11B65, 11F11, 11F20, 33C05.

Key words and phrases. Theta functions, $q$-series, eta function, modular forms, cubic modular equations, hypergeometric functions.

Research of the first and second author was partially supported by NSERC.

Research completed while the third author was an NSERC International Fellow. 
and the Jacobian theta functions

$$
\begin{aligned}
& \theta_{2}(q):=\sum_{n=-\infty}^{\infty} q^{\left(n+\frac{1}{2}\right)^{2}}, \\
& \theta_{3}(q):=\sum_{n=-\infty}^{\infty} q^{n^{2}},
\end{aligned}
$$

and

$$
\theta_{4}(q):=\sum_{n=-\infty}^{\infty}(-1)^{n} q^{n^{2}}
$$

Jacobi's quartic identity, $\theta_{3}^{4}=\theta_{4}^{4}+\theta_{2}^{4}$, plays a key role in theta function and elliptic function theory $[2,4]$. The cubic identity $(1.1)$. has very similar consequences which are detailed in [5]. Let us denote

$$
\begin{aligned}
& a(q):=\sum_{n, m=-\infty}^{\infty} q^{n^{2}+n m+m^{2}}, \\
& b(q):=\sum_{n, m=-\infty}^{\infty} \omega^{n-m} q^{n^{2}+n m+m^{2}}, \\
& c(q):=\sum_{n, m=-\infty}^{\infty} q^{\left(n+\frac{1}{3}\right)^{2}+\left(n+\frac{1}{3}\right)\left(m+\frac{1}{3}\right)+\left(m+\frac{1}{3}\right)^{2}} .
\end{aligned}
$$

We will prove that $a^{3}=b^{3}+c^{3}$. Note that in (1.3) and (1.8) we take principal roots.

\section{Results}

Lemma 2.1. For $|q|<1$

(i) (a) $a(q)=\theta_{3}(q) \theta_{3}\left(q^{3}\right)+\theta_{2}(q) \theta_{2}\left(q^{3}\right)$;

(b) $a\left(q^{4}\right)=\frac{1}{2}\left(\theta_{3}(q) \theta_{3}\left(q^{3}\right)+\theta_{4}(q) \theta_{4}\left(q^{3}\right)\right)$;

(ii) $b(q)=\frac{3}{2} a\left(q^{3}\right)-\frac{1}{2} a(q)$;

(iii) $c(q)=\frac{1}{2} a\left(q^{\frac{1}{3}}\right)-\frac{1}{2} a(q)$.

Proof. (i) In (1.6) write $n^{2}+n m+m^{2}=\left(n+\frac{m}{2}\right)^{2}+3\left(\frac{m}{2}\right)^{2}$. Now

$$
a(q)=\sum_{m \text { even }} q^{\left(n+\frac{m}{2}\right)^{2}+3\left(\frac{m}{2}\right)^{2}}+\sum_{m \text { odd }} q^{\left(n+\frac{m}{2}\right)^{2}+3\left(\frac{m}{2}\right)^{2}} .
$$

This identity is (i) (a). Identity (i) (b) follows from $\theta_{3}\left(q^{4}\right)+\theta_{2}\left(q^{4}\right)=\theta_{3}(q)$ and $\theta_{3}\left(q^{4}\right)-\theta_{2}\left(q^{4}\right)=\theta_{4}(q)$.

(ii) We observe that $b(q)$ is real and that

$$
\begin{aligned}
b(q) & =\sum \cos \left[\frac{2 \pi}{3}(n-m)\right] q^{n^{2}+n m+m^{2}} \\
& =\frac{3}{2} \sum_{3 \mid n-m} q^{n^{2}+n m+m^{2}}-\frac{1}{2} \sum q^{n^{2}+n m+m^{2}} .
\end{aligned}
$$

Now we write the first sum by

$$
\sum_{m} \sum_{p} q^{(3 p+m)^{2}+(3 p+m) m+m^{2}}=a\left(q^{3}\right),
$$


as is apparent on substituting $m-p$ for $m$. Identity (ii) follows.

(iii) Since (ii) holds, it suffices to show that

$$
c\left(q^{3}\right)=\frac{1}{3}(a(q)-b(q)) .
$$

Now the right-hand side of $(2.1)$ is

$$
\frac{1}{2} \sum_{3 \nmid n-m} q^{n^{2}+n m+m^{2}}=\sum_{n-m \equiv 1(\bmod 3)} q^{n^{2}+n m+m^{2}}=\sum_{m, p=-\infty}^{\infty} q^{(m+3 p+1)^{2}+(m+3 p+1) m+m^{2}} .
$$

On replacing $m$ by $m-p$ (bijectively) we have

$$
\frac{1}{3}(a(q)-b(q))=\sum_{p, m} q^{3 p^{2}+3 p+3 p m+3 m^{2}+3 m+1},
$$

which, by (1.8), equals $c\left(q^{3}\right)$. Thus, (2.1) has been proved.

As is well known, [4, p. 64], the theta functions possess simple product expansions. This is not true of $a$. It is true of $b$ and $c$ as our main preliminary result shows:

\section{Proposition 2.2.}

$$
\begin{aligned}
& b(q)=\frac{\eta^{3}(q)}{\eta\left(q^{3}\right)}=\prod_{n \geq 1} \frac{\left(1-q^{n}\right)^{3}}{\left(1-q^{3 n}\right)} \\
& c(q)=3 \frac{\eta^{3}\left(q^{3}\right)}{\eta(q)}=3 q^{\frac{1}{3}} \prod_{n \geq 1} \frac{\left(1-q^{3 n}\right)^{3}}{\left(1-q^{n}\right)}
\end{aligned}
$$

Proof. (i) We need the following result due to Euler (which is a corollary of the $q$-binomial theorem [1, p. 19], [4, p. 309]). We use

$$
(-x ; q)_{\infty}=\prod_{n=0}^{\infty}\left(1+x q^{n}\right)=\sum_{k=0}^{\infty} \frac{q^{\left(\begin{array}{l}
k \\
2
\end{array}\right)} x^{k}}{(q)_{k}}
$$

where as usual

$$
\begin{aligned}
(a)_{\infty} & =(a ; q)_{\infty}:=\prod_{n=0}^{\infty}\left(1-a q^{n}\right), \\
(a)_{n} & =(a ; q)_{n}:=(a ; q)_{\infty} /\left(a q^{n} ; q\right)_{\infty}=\prod_{k=1}^{n}\left(1-a q^{k-1}\right) .
\end{aligned}
$$

Observe that

$$
\left(-x^{3} ; q^{3}\right)_{\infty}=(-x ; q)_{\infty}(-x \omega ; q)_{\infty}\left(-x \omega^{2} ; q\right)_{\infty}
$$

so that (2.2) gives

$$
\sum_{k=0}^{\infty} \frac{x^{3 k} q^{3\left(\begin{array}{l}
k \\
2
\end{array}\right)}}{\left(q^{3} ; q^{3}\right)_{k}}=\sum_{n_{0}, n_{1}, n_{2} \geq 0} \omega^{n_{1}+2 n_{2}} \frac{q^{\left(\begin{array}{c}
n_{0} \\
2
\end{array}\right)+\left(\begin{array}{c}
n_{1} \\
2
\end{array}\right)+\left(\begin{array}{c}
n_{2} \\
2
\end{array}\right)} x^{n_{0}+n_{1}+n_{2}}}{(q)_{n_{0}}(q)_{n_{1}}(q)_{n_{2}}}
$$

Equating coefficients of powers of $x$ yields

$$
\frac{1}{\left(q^{3} ; q^{3}\right)_{k}}=\sum_{n_{0}+n_{1}+n_{2}=3 k} \omega^{n_{1}-n_{2}} \frac{q^{\left(\begin{array}{c}
n_{0} \\
2
\end{array}\right)+\left(\begin{array}{c}
n_{1} \\
2
\end{array}\right)+\left(\begin{array}{c}
n_{2} \\
2
\end{array}\right)-3\left(\begin{array}{c}
k \\
2
\end{array}\right)}}{(q)_{n_{0}}(q)_{n_{1}}(q)_{n_{2}}}
$$


We replace $n_{i}$ by $m_{i}+k$ for $i=0,1,2$. Then

$$
\sum_{i=0}^{2}\left(\begin{array}{c}
n_{i} \\
2
\end{array}\right)=\frac{1}{2} \sum_{i=0}^{2} m_{i}^{2}+3\left(\begin{array}{l}
k \\
2
\end{array}\right)
$$

since $\sum_{i=0}^{2} m_{i}=0$. Hence

$$
\frac{1}{\left(q^{3} ; q^{3}\right)_{k}}=\sum_{m_{0}+m_{1}+m_{2}=0} \omega^{m_{1}-m_{2}} \frac{q^{\frac{1}{2}\left(m_{0}^{2}+m_{1}^{2}+m_{2}^{2}\right)}}{(q)_{m_{0}+k}(q)_{m_{1}+k}(q)_{m_{2}+k}} .
$$

Letting $k$ tend to infinity gives

$$
\frac{1}{\left(q^{3} ; q^{3}\right)_{\infty}}=\sum_{m_{0}+m_{1}+m_{2}=0} \omega^{m_{1}-m_{2}} \frac{q^{\frac{1}{2}\left(m_{0}^{2}+m_{1}^{2}+m_{2}^{2}\right)}}{(q)_{\infty}^{3}}
$$

Hence

$$
\begin{aligned}
\frac{(q)_{\infty}^{3}}{\left(q^{3} ; q^{3}\right)_{\infty}} & =\sum_{m_{0}+m_{1}+m_{2}=0} \omega^{m_{1}-m_{2}} q^{\frac{1}{2}\left(m_{0}^{2}+m_{1}^{2}+m_{2}^{2}\right)} \\
& =\sum_{m_{1}, m_{2}} \omega^{m_{1}-m_{2}} q^{m_{1}^{2}+m_{1} m_{2}+m_{2}^{2}}
\end{aligned}
$$

as claimed.

(ii) This may be established in a similar fashion. Alternatively, we may argue as follows. The previous identity combines with Lemma 2.1(ii) to produce

$$
\frac{\eta^{3}(q)}{\eta\left(q^{3}\right)}=\frac{3 a\left(q^{3}\right)-a(q)}{2}
$$

The theta transformation formulae [4, p. 38]

$$
\sqrt{t} \theta_{3}\left(e^{-\pi t}\right)=\theta_{3}\left(e^{-\pi / t}\right), \quad \sqrt{t} \theta_{4}\left(e^{-\pi t}\right)=\theta_{2}\left(e^{-\pi / t}\right)
$$

may be applied to $a$ as given by Lemma 2.1(i) (a). The corresponding transformation of $\eta$,

$$
\sqrt{t} \eta\left(e^{-2 \pi t}\right)=\eta\left(e^{-2 \pi / t}\right)
$$

and Lemma 2.1 (i) (b) yields

$$
3 \frac{\eta^{3}\left(q^{3}\right)}{\eta(q)}=\frac{a\left(q^{\frac{1}{3}}\right)-a(q)}{2}
$$

Now Lemma 2.1 (iii) completes the proof.

We are now ready to establish identity (1.1) which, as we have seen, is equivalent to

\section{Theorem 2.3.}

$$
a^{3}(q)=b^{3}(q)+c^{3}(q)
$$

Proof. Let us set $a^{*}:=a\left(q^{3}\right), b^{*}:=b\left(q^{3}\right), c^{*}:=c\left(q^{3}\right)$. Now Lemma 2.1 shows $a^{*}-c^{*}=b$. Hence

$$
b(q) b(\omega q) b\left(\omega^{2} q\right)=\left(a^{*}-c^{*}\right)\left(a^{*}-\omega c^{*}\right)\left(a^{*}-\omega^{2} c^{*}\right),
$$


since $a^{*}\left(\omega^{i} q\right)=a^{*}$ and $c^{*}\left(\omega^{i} q\right)=\omega^{i} c^{*}$. Thus

$$
a^{* 3}-c^{* 3}=\prod_{i=0}^{2} b\left(\omega^{i} q\right)=b^{* 3},
$$

where the last identity is an easy consequence of Proposition 2.2 (i). On replacing $q^{3}$ by $q$ we are done.

For completeness we record the following corollary established in [5].

\section{Corollary 2.4.}

$$
{ }_{2} F_{1}\left(\frac{1}{3}, \frac{2}{3} ; 1 ; \frac{c^{3}(q)}{a^{3}(q)}\right)=a(q) .
$$

The crucial step in establishing (2.5) involves determining what happens as $q \rightarrow q^{3}$ on both sides of (2.5). Armed with Theorem 2.3 and Lemma 2.1, this reduces to establishing that $F(s):={ }_{2} F_{1}\left(\frac{1}{3}, \frac{2}{3} ; 1 ; s^{3}\right)$ satisfies

$$
F\left(\frac{c^{*}}{a^{*}}\right)=\left(\frac{1+2 \frac{b}{a}}{3}\right) F\left(\frac{c}{a}\right) .
$$

Since $\frac{c^{*}}{a^{*}}=\frac{(1-b / a)}{(1+2 b / a)}$, this becomes a cubic transformation for $F$, and can be verified in various ways (including symbolically). Details of the derivation of this cubic transformation may be found in [6].

Next we observe that the classical cubic modular equation for $\eta$ is easily accessible from Theorem 2.3. This modular equation is Entry 1(iv) of Chapter 20 in Ramanujan's second notebook [3, p. 345].

\section{Corollary 2.5.}

$$
\left[1+9 \frac{\eta^{3}\left(q^{9}\right)}{\eta^{3}(q)}\right]^{3}=1+27\left[\frac{\eta\left(q^{3}\right)}{\eta(q)}\right]^{12}
$$

Proof. Let $t:=a / b$ and note as above that

$$
1+\left(\frac{b^{*}}{c^{*}}\right)^{3}=\left(\frac{a^{*}}{c^{*}}\right)^{3}=\left(\frac{t+2}{t-1}\right)^{3}
$$

Thus

$$
\left(\frac{c^{*}}{b^{*}}\right)^{3}=\frac{(t-1)^{4}}{9\left(t^{3}-1\right)}
$$

while

$$
\left(\frac{c}{b}\right)^{3}=t^{3}-1
$$

Now use Proposition 2.2 to write

$$
\frac{c}{b}=3 \frac{\eta^{4}\left(q^{3}\right)}{\eta^{4}(q)}
$$

Finally (2.7)-(2.9) combine to yield

$$
(t-1)^{4}=9\left(\frac{c c^{*}}{b b^{*}}\right)^{3}=\left[9 \frac{\eta^{3}\left(q^{9}\right)}{\eta^{3}(q)}\right]^{4}
$$


Hence

$$
\left[1+9 \frac{\eta^{3}\left(q^{9}\right)}{\eta^{3}(q)}\right]^{3}=1+\left(\frac{c}{b}\right)^{3}=1+27\left[\frac{\eta\left(q^{3}\right)}{\eta(q)}\right]^{12} .
$$

We observe that in terms of the eta-multiplier, $N_{3}:=\eta^{2}\left(q^{3}\right) / \eta^{2}(q)$, (2.6) becomes

$$
N_{3}\left(q^{3}\right)=\frac{\left[\left(1+27 N_{3}^{6}\right)^{\frac{1}{3}}-1\right]^{\frac{2}{3}}}{3^{\frac{4}{3}} N_{3}} .
$$

The parallel quintic and septic identities, due to Ramanujan, are solvable and are given in [4, p. 312].

We now prove the corresponding quadratic modular equation given by Ramanujan. It is the first equation on page 259 of the second notebook [12].

Theorem 2.6. For $|q|<1$

$$
a(q) a\left(q^{2}\right)=b(q) b\left(q^{2}\right)+c(q) c\left(q^{2}\right)
$$

or equivalently

$$
(1-u v)^{3}=\left(1-u^{3}\right)\left(1-v^{3}\right)
$$

where $u:=c(q) / a(q)$ and $v:=c\left(q^{2}\right) / a\left(q^{2}\right)$.

Proof. (i) Let

$$
L(q):=a(q) a\left(q^{2}\right)-b(q) b\left(q^{2}\right)-c(q) c\left(q^{2}\right) .
$$

We will show that

$$
L(q)=L(-q)
$$

It will follow that

$$
\begin{aligned}
L(q)=\frac{L(q)+L(-q)}{2}= & \frac{a(q)+a(-q)}{2} a\left(q^{2}\right) \\
& -\frac{b(q)+b(-q)}{2} b\left(q^{2}\right)-\frac{c(q)+c(-q)}{2} c\left(q^{2}\right)
\end{aligned}
$$

However, since $\theta_{2}(q) \theta_{2}\left(q^{3}\right)$ is an odd function we have

$$
\frac{a(q)+a(-q)}{2}=\frac{\theta_{3}(q) \theta_{3}\left(q^{3}\right)+\theta_{4}(q) \theta_{4}\left(q^{3}\right)}{2}=a\left(q^{4}\right)
$$

by Lemma (2.1) (i) (b). Lemma 2.1 (ii) and (iii) now show that

$$
\frac{b(q)+b(-q)}{2}=b\left(q^{4}\right) \text { and } \frac{c(q)+c(-q)}{2}=c\left(q^{4}\right) .
$$

Hence (2.11) becomes $L(q)=L\left(q^{2}\right)$. Inductively, $L(q)=L\left(q^{2^{n}}\right)$ and hence $L(q)=L(0)=0$ as claimed.

To establish (2.10) we define

$$
x(q):=\frac{b(q)-b(-q)}{a(q)-a(-q)}, \quad y(q):=\frac{c(q)-c(-q)}{a(q)-a(-q)},
$$

and note that $(2.10)$ is equivalent to

$$
a\left(q^{2}\right)=x(q) b\left(q^{2}\right)+y(q) c\left(q^{2}\right)
$$


Now

$$
\begin{aligned}
a(q)-a(-q) & =\left[\theta_{3}(q) \theta_{3}\left(q^{3}\right)+\theta_{2}(q) \theta_{2}\left(q^{3}\right)\right]-\left[\theta_{4}(q) \theta_{4}\left(q^{3}\right)-\theta_{2}(q) \theta_{2}\left(q^{3}\right)\right] \\
& =3 \theta_{2}(q) \theta_{2}\left(q^{3}\right)
\end{aligned}
$$

on applying the cubic modular equation for $\theta_{2}^{2} / \theta_{3}^{2}[4$, p. 110]. Thus Lemma 2.1 (ii) yields the first equation in the following.

$$
2 x(q)=3 \frac{\theta_{2}\left(q^{9}\right)}{\theta_{2}(q)}-1=-\prod_{n=1}^{\infty} \frac{\left(1+q^{6 n}\right)}{\left(1+q^{2 n}\right)^{3}} .
$$

The second equation follows from [4, Theorem 4.11 (c)] which is proved using the quintuple product identity. Here we a give a proof using Jacobi's triple product identity $[4,(3.1 .1)$, p. 62$]$ :

$$
\sum_{n=-\infty}^{\infty}(-1)^{n} z^{n} q^{n^{2}}=\prod_{n=1}^{\infty}\left(1-q^{2 n}\right)\left(1-z q^{2 n-1}\right)\left(1-z^{-1} q^{2 n-1}\right)
$$

Now,

$$
\begin{aligned}
\theta_{4}(q) & =\sum_{n=-\infty}^{\infty}(-1)^{n} q^{n^{2}} \\
& =\sum_{n=-\infty}^{\infty}(-1)^{n} q^{(3 n)^{2}}-\sum_{n=-\infty}^{\infty}(-1)^{n} q^{(3 n+1)^{2}}-\sum_{n=-\infty}^{\infty}(-1)^{n} q^{(3 n-1)^{2}} \\
& =\theta_{4}\left(q^{9}\right)-2 q \sum_{n=-\infty}^{\infty}(-1)^{n} q^{9 n^{2}+6 n}
\end{aligned}
$$

Using (2.15) we find, after some manipulation, that

$$
\begin{aligned}
\theta_{4}(q)-\theta_{4}\left(q^{9}\right) & =-2 q \sum_{n=-\infty}^{\infty}(-1)^{n} q^{9 n^{2}+6 n} \\
& =-2 q \prod_{n=1}^{\infty}\left(1-q^{18 n}\right)\left(1-q^{18 n-3}\right)\left(1-q^{18 n-15}\right) \\
& =-2 \frac{\eta\left(q^{3}\right) \eta^{2}\left(q^{18}\right)}{\eta\left(q^{6}\right) \eta\left(q^{9}\right)}
\end{aligned}
$$

Now, replace $q$ by $e^{-\pi t}$, apply the transformation $t \rightarrow \frac{1}{9 t}$, and use the transformation formulae:

$$
\eta\left(e^{-\pi / t}\right)=\sqrt{2 t} \eta\left(e^{-4 \pi t}\right), \quad \theta_{4}\left(e^{-\pi / t}\right)=\sqrt{t} \theta_{2}\left(e^{-\pi t}\right) .
$$

We find after some simplification, that

$$
3 \theta_{2}\left(q^{9}\right)-\theta_{2}(q)=-2 \frac{\eta\left(q^{12}\right) \eta^{2}\left(q^{2}\right)}{\eta\left(q^{6}\right) \eta\left(q^{4}\right)} .
$$

Since $\theta_{2}(q)=2 \eta^{2}\left(q^{4}\right) / \eta\left(q^{2}\right)[4$, p. 64] we obtain the second equation in (2.14) after dividing both sides by $\theta_{2}(q)$. In consequence, Proposition 2.2 (i) gives

$$
2 x(q) b\left(q^{2}\right)=-\prod_{n=1}^{\infty}\left(\frac{1-q^{2 n}}{1+q^{2 n}}\right)^{3} / \prod_{n=1}^{\infty}\left(\frac{1-q^{6 n}}{1+q^{6 n}}\right) .
$$


But $\prod_{n=1}^{\infty}\left(1-q^{n}\right) /\left(1+q^{n}\right)=\theta_{4}(q)[4$, p. 64]. Thus

$$
2 x(q) b\left(q^{2}\right)=-\theta_{4}\left(q^{2}\right)^{3} / \theta_{4}\left(q^{6}\right) \text {. }
$$

Similarly, we find that

$$
2 y(q)=\frac{\theta_{2}\left(q^{\frac{1}{3}}\right)}{\theta_{2}\left(q^{3}\right)}-1=q^{-\frac{2}{3}} \prod_{n=1}^{\infty} \frac{\left(1+q^{2 n}\right)}{\left(1+q^{6 n}\right)^{3}} .
$$

The proof of the first equation in (2.18) is analogous to that of the first equation in (2.14) and uses Lemma 2.1 (iii). The proof of the second equation is analogous to that of (2.16). It also follows from [4, Theorem 4.11 (c)]. Let

$$
\theta_{2}^{*}(q)=\sum_{n=-\infty}^{\infty} q^{n^{2}+n}, \quad \text { so that } \quad \theta_{2}(q)=q^{\frac{1}{4}} \theta_{2}^{*}(q)
$$

Replacing $n$ by $3 m+k(k=0, \pm 1)$ in the summation yields

$$
\theta_{2}^{*}(q)=q^{2} \theta_{2}^{*}\left(q^{9}\right)+2 \sum_{m=-\infty}^{\infty} q^{9 m^{2}+3 m}
$$

So, by (2.15), we have

$$
\begin{aligned}
\theta_{2}^{*}\left(q^{\frac{1}{3}}\right)-q^{\frac{2}{3}} \theta_{2}^{*}\left(q^{3}\right) & =2 \sum_{m=-\infty}^{\infty} q^{3 m^{2}+m} \\
& =2 \prod_{n=1}^{\infty}\left(1-q^{6 n}\right)\left(1+q^{6 n-2}\right)\left(1-q^{6 n-4}\right) \\
& =2 \prod_{n=1}^{\infty} \frac{\left(1-q^{6 n}\right)\left(1+q^{2 n}\right)}{\left(1+q^{6 n}\right)} \\
& =\prod_{n=1}^{\infty} \frac{\left(1+q^{2 n}\right)}{\left(1+q^{6 n}\right)^{3}}\left(2 \prod_{n=1}^{\infty} \frac{\left(1-q^{12 n}\right)^{2}}{\left(1-q^{6 n}\right)}\right)
\end{aligned}
$$

and

$$
\frac{\theta_{2}^{*}\left(q^{\frac{1}{3}}\right)}{\theta_{2}^{*}\left(q^{3}\right)}-q^{\frac{2}{3}}=\prod_{n=1}^{\infty} \frac{\left(1+q^{2 n}\right)}{\left(1+q^{6 n}\right)^{3}} .
$$

After multiplying both sides by $q^{-2 / 3}$ we obtain the second equation in (2.18). Using Proposition 2.2 (ii) we have

$$
2 y(q) c\left(q^{2}\right)=3 \prod_{n=1}^{\infty}\left(\frac{1-q^{6 n}}{1+q^{6 n}}\right)^{3} / \prod_{n=1}^{\infty}\left(\frac{1-q^{2 n}}{1+q^{2 n}}\right)
$$

whence

$$
2 y(q) c\left(q^{2}\right)=3 \theta_{4}\left(q^{6}\right)^{3} / \theta_{4}\left(q^{2}\right)
$$

Thus (2.10) is equivalent to

$$
a(q)=\frac{3}{2} \frac{\theta_{4}^{3}\left(q^{3}\right)}{\theta_{4}(q)}-\frac{1}{2} \frac{\theta_{4}^{3}(q)}{\theta_{4}\left(q^{3}\right)} .
$$


Here we have simplified (2.13), the equation equivalent to (2.10), using (2.17) and (2.19). We need the Lambert series for $a(q)$ :

$$
a(q)=1+6 \sum_{n=0}^{\infty}\left\{\frac{q^{3 n+1}}{1-q^{3 n+1}}-\frac{q^{3 n+2}}{1-q^{3 n+2}}\right\} .
$$

This identity is due to Lorenz [11, p. 111]. The referee has informed us Ramanujan had a proof of this result; see his Saturday night letter from Fitzroy house [13, pp. 93-96]. The referee also states that it can be proved by combining together some results found in Ramanujan's notebooks. It also follows from Kolitsch [10, Lemma 2]. We now sketch how (2.21) follows from Lemma 2.1 (i)(a), [4, Ex.5(ii), p. 287] and the cubic modular equation [4, (4.2.7), p. 110]. From [4, Ex.5(ii), p. 287] we have

$$
\begin{aligned}
\theta_{3}(q) \theta_{3}\left(q^{3}\right)= & 1+2 \sum_{n=1}^{\infty} \frac{\left(1-q^{n}\right) q^{n}}{\left(1-q^{3 n}\right)}+4 \sum_{n=1}^{\infty} \frac{\left(1-q^{4 n}\right) q^{4 n}}{\left(1-q^{12 n}\right)} \\
= & 1+2 \sum_{n=1}^{\infty} \frac{\left(1-q^{2 n}\right) q^{2 n}}{\left(1-q^{6 n}\right)}+2 \sum_{\substack{n=1 \\
n \text { odd }}}^{\infty} \frac{\left(1-q^{n}\right) q^{n}}{\left(1-q^{3 n}\right)} \\
& +4 \sum_{n=1}^{\infty} \frac{\left(1-q^{4 n}\right) q^{4 n}}{\left(1-q^{12 n}\right)} .
\end{aligned}
$$

By replacing $q$ by $-q$ we find after some simplification that

$$
\begin{aligned}
\theta_{4}(q) \theta_{4}\left(q^{3}\right)= & +2 \sum_{n=1}^{\infty} \frac{\left(1-q^{2 n}\right) q^{2 n}}{\left(1-q^{6 n}\right)}-2\left\{\sum_{n=1}^{\infty} \frac{\left(1+q^{n}\right) q^{n}}{\left(1+q^{3 n}\right)}-\sum_{n=1}^{\infty} \frac{\left(1+q^{2 n}\right) q^{2 n}}{\left(1+q^{6 n}\right)}\right\} \\
& +4 \sum_{n=1}^{\infty} \frac{\left(1-q^{4 n}\right) q^{4 n}}{\left(1-q^{12 n}\right)} \\
= & 1-2 \sum_{n=1}^{\infty} \frac{\left(1-q^{4 n}\right) q^{n}}{\left(1-q^{6 n}\right)}+2 \sum_{n=1}^{\infty} \frac{\left(1-q^{4 n}\right)\left(1+3 q^{2 n}+q^{4 n}\right) q^{2 n}}{\left(1-q^{12 n}\right)} .
\end{aligned}
$$

From Lemma 2.1 (i)(a) and [4, (4.2.7) p. 110] we have

$$
\begin{aligned}
a(q) & =\theta_{3}(q) \theta_{3}\left(q^{3}\right)+\theta_{2}(q) \theta_{2}\left(q^{3}\right) \\
& =2 \theta_{3}(q) \theta_{3}\left(q^{3}\right)-\theta_{4}(q) \theta_{4}\left(q^{3}\right) .
\end{aligned}
$$

After rewriting (2.24) using (2.22) and (2.23) we find after much simplification that

$$
\begin{aligned}
a(q) & =1+6 \sum_{n=1}^{\infty} \frac{\left(1-q^{n}\right) q^{n}}{\left(1-q^{3 n}\right)} \\
& =1+6 \sum_{n=1}^{\infty} \sum_{m=0}^{\infty} q^{n(3 m+1)}-q^{n(3 m+2)},
\end{aligned}
$$

and (2.21) follows by reversing the order of summation. 
The following result is Entry 4(iv) in Chapter 19 of Ramanujan's second notebook [3, p. 227]. Ewell [7; 4, p. 151] has also given a proof using the quintuple product identity.

$$
\frac{\theta_{4}^{3}(q)}{\theta_{4}\left(q^{3}\right)}=1-6 \sum_{n=0}^{\infty}\left\{\frac{q^{3 n+1}}{1+q^{3 n+1}}-\frac{q^{3 n+2}}{1+q^{3 n+2}}\right\} .
$$

Hence we have

$$
\frac{\theta_{4}^{3}(q)}{\theta_{4}\left(q^{3}\right)}=2 a\left(q^{2}\right)-a(q)
$$

This can also be deduced from Fine [8, (32.64), p. 84]. Dually, on replacing $q$ by $-q$, using the theta transform and replacing $q$ by $-q$ again

$$
\frac{\theta_{4}^{3}\left(q^{3}\right)}{\theta_{4}(q)}=\frac{1}{3} a(q)+\frac{2}{3} a\left(q^{2}\right)
$$

This can also be deduced from Fine $[8,(32.39)$, p. 80]. Now (2.26) and (2.27) combine to establish (2.20) and so (2.10).

(ii) Write (i) as

$$
1-u v=\frac{b(q)}{a(q)} \frac{b\left(q^{2}\right)}{a\left(q^{2}\right)}
$$

and cube both sides. Then Theorem 2.3 produces

$$
(1-u v)^{3}=\frac{b^{3}(q) b^{3}\left(q^{2}\right)}{a^{3}(q) a^{3}\left(q^{2}\right)}=\left(1-u^{3}\right)\left(1-v^{3}\right) .
$$

Note that (ii) is Ramanujan's preferred form. We also note that

$$
2 x(q)=-b\left(q^{2}\right) / b\left(q^{4}\right) \text { and } 2 y(q)=c\left(q^{2}\right) / c\left(q^{4}\right),
$$

so that

$$
a(q)=\frac{1}{2} \frac{c^{2}(q)}{c\left(q^{2}\right)}-\frac{1}{2} \frac{b^{2}(q)}{b\left(q^{2}\right)}
$$

\section{THE MODULAR MACHINE}

It is possible to both find and prove the two key identities of this note, (1.1) and Theorem 2.6 (i), entirely mechanically. This works as follows. From (2.21) we see that $a(q)$ (in the variable $\tau, q=e^{2 \pi i \tau}$ ) is an Eisenstein series of weight one and character $\chi(d)=\left(\frac{d}{3}\right)$ (the Legendre symbol modulo 3 ) for the congruence subgroup $\Gamma_{0}(3)$. See [9, p. 4]. It is well known that if $f(\tau)$ is a modular form on $\Gamma_{0}(N)$ then $f(M \tau)$ is a modular form on $\Gamma_{0}(N M)$. From Lemma 2.1 (ii), (iii) it follows that $a\left(q^{3}\right), b\left(q^{3}\right), c\left(q^{3}\right), a\left(q^{6}\right), b\left(q^{6}\right)$, $c\left(q^{6}\right)$ are all entire modular forms of weight one (and trivial character) on some congruence subgroup $G$ where

$$
\Gamma(3) \cap \Gamma_{0}(54) \subset G \subset \Gamma
$$

(we are working in the variable $q^{3}$ to give $c$ a Taylor series expansion at $i \infty$ ). 
Here as usual

$$
\begin{aligned}
\Gamma & :=\left\{\left(\begin{array}{ll}
\alpha & \beta \\
\gamma & \delta
\end{array}\right) \mid \alpha \delta-\beta \gamma=1, \quad \alpha, \beta, \gamma, \delta \in \mathbb{Z}\right\}, \\
\Gamma(N) & :=\left\{\left(\begin{array}{ll}
\alpha & \beta \\
\gamma & \delta
\end{array}\right) \in \Gamma \mid \alpha \equiv \delta \equiv 1 \text { and } \beta \equiv \gamma \equiv 0 \bmod N\right\}, \\
\Gamma_{0}(N) & :=\left\{\left(\begin{array}{ll}
\alpha & \beta \\
\gamma & \delta
\end{array}\right) \in \Gamma \mid \gamma \equiv 0 \bmod N\right\} .
\end{aligned}
$$

The indices satisfy

$$
[\Gamma: \Gamma(N)]=N^{3} \prod_{p \mid N}\left(1-\frac{1}{p^{2}}\right)
$$

and

$$
\left[\Gamma: \Gamma_{0}(N)\right]=N \prod_{p \mid N}\left(1+\frac{1}{p}\right)
$$

It follows that

$$
[\Gamma: G] \leq[\Gamma: \Gamma(3)] \cdot\left[\Gamma: \Gamma_{0}(54)\right] \leq 24 \cdot 108 .
$$

(This standard theory is in [14].)

Now suppose

$$
P:=P(a, b, c, A, B, C)
$$

is a homogeneous polynomial of degree $N$ in the 6 variables $a:=a(q), b:=$ $b(q), c:=c(q), A:=a\left(q^{2}\right), B:=b\left(q^{2}\right), C:=c\left(q^{2}\right)$. Then $P(q)$ is an entire modular form of weight $N$ on $G$ and hence can have exactly $N[\Gamma: G] / 12$ zeros in a fundamental region (counted in the local variables at the cusps). In particular $P$ can have a zero of order at most

$$
\frac{N[\Gamma: G]}{12} \leq 216 N
$$

at $\tau=i \infty$. In other words if the $q$-expansion of $P$ vanishes through the first $216 N+1$ terms then $P \equiv 0$.

It is now a straightforward matter to generate a basis for all homogeneous identities of type (3.1) for a fixed $N$. One expands the six functions $a, b, c, A$, $B, C$ as $q$-series to some fixed order that is greater than the number of monomials in the expansion of $\left(x_{1}+x_{2}+x_{3}+x_{4}+x_{5}+x_{6}\right)^{N}$. One then solves the linear problem of finding a basis of identities to this fixed order. This must now be a superset of the desired identities. One then verifies that the $q$-expansion of each basis element vanishes through $216 N+1$ terms; which proves that the alleged identity is a true identity (and not just an identity to a fixed number of terms). Since this is all done in exact integer arithmetic in a symbolic manipulation package this constitutes both a derivation and a proof.

We illustrate with $N=3$. What follows is a basis for all homogeneous cubic relations in $a:=a(q), b:=b(q), c:=c(q), A:=a\left(q^{2}\right), B:=b\left(q^{2}\right)$, $C:=c\left(q^{2}\right)$. 

(1*) $A\left(c^{2}-a C-2 A C\right)$
$\left(17^{*}\right)-C^{3}-B^{3}+A^{3}$
(2) $B\left(c^{2}-a C-2 A C\right)$
(18) $4 C^{3}-3 a c C+c^{3}$
(3*) $\quad c(b B-A a+c C)$
$\left(19^{*}\right) \quad 2 B^{3}-3 b A B+b^{3}$
(4) $\quad B(b B-A a+c C)$
$\left(20^{*}\right)-b c A-b C^{2}+c B^{2}$
(5) $\quad C(b B-A a+c C)$
(21) $\quad b c C-a B^{2}+b A^{2}$
(6) $\quad A\left(c A-a c+2 C^{2}\right)$
(22) $b c^{2}-b A C-2 B^{2} C$
(7) $B\left(c A-a c+2 C^{2}\right)$
(23) $b^{2} c-a c B+4 B C^{2}$
(8) $C\left(c A-a c+2 C^{2}\right)$
(24) $a b^{2}-a A B-2 b B^{2}+2 A^{2} B$
(9) $-b\left(c A-a c+2 C^{2}\right)$
(25) $b c C+a^{2} b-3 a B^{2}+2 A B^{2}$
(10) $B\left(b A-2 B^{2}+a b\right)$
(26) $2 b B C-3 a A C+a c^{2}-2 A^{2} C$
(11*) $C\left(b A-2 B^{2}+a b\right)$
$\left(12^{*}\right)-A\left(-a B-b^{2}+2 A B\right)$
(28) $a b A-b c C+a B^{2}-2 A B^{2}$
(13) $-B\left(-a B-b^{2}+2 A B\right)$
(29) $b c B-a c A+a C^{2}+2 A C^{2}$
(14) $-C\left(-a B-b^{2}+2 A B\right)$
(30) $b A B-a c C-2 B^{3}+a^{2} A$
(15) $-B\left(A a-2 b B-a^{2}+2 A^{2}\right)$
(31) $-b A B-a c C+2 C^{3}+a A^{2}$
(16) $-C\left(A a-2 b B-a^{2}+2 A^{2}\right)$
(32) $-3 b A B-3 a c C+4 C^{3}+2 B^{3}+a^{3}$

We find 32 of them, presented in factored form. Since there is a basis of six quadratic relations many of them factor. Relations are starred if they correspond to identities obtained earlier in this paper, or if they are needed in the proof of the results given below. The verification of the identities requires computing Taylor series of length 650 . The entire calculation takes just a few minutes in MAPLE on a SUN4. Note that basis element $\left(17^{*}\right)$ is our cubic modular equation while basis element $\left(3^{*}\right)$ is our quadratic identity. More thought would allow checking to a lower degree in the $q$-expansion, however since the computations are easy we have opted for the most straightforward estimates. Similar remarks apply for other forms and other $N$. Note that $\left(1^{*}\right)$ and $\left(12^{*}\right)$ combine to verify $(2.28)$

while $\left(20^{*}\right)$ is

$$
a=\frac{1}{2} \frac{c^{2}}{C}-\frac{1}{2} \frac{b^{2}}{B},
$$

and $\left(19^{*}\right)$ is

$$
A=\frac{B^{2}}{b}-\frac{C^{2}}{c}
$$

$$
A=\frac{2}{3} \frac{B^{2}}{b}+\frac{1}{3} \frac{b^{2}}{B}
$$

From $\left(11^{*}\right)$ and $\left(12^{*}\right)$ we may solve for $A, B$. After some work we find we get

$$
a\left(q^{2}\right)=A=\operatorname{Re}\left(b^{\frac{3}{2}}+i c^{\frac{3}{2}}\right)^{\frac{2}{3}}=a \cos \left(\frac{2}{3} \arctan \left(\frac{c}{b}\right)^{\frac{3}{2}}\right),
$$




$$
b\left(q^{2}\right)=B=\operatorname{Re}\left(b^{3}+i b^{\frac{3}{2}} c^{\frac{3}{2}}\right)^{\frac{1}{3}}=\sqrt{a b} \cos \left(\frac{1}{3} \arctan \left(\frac{c}{b}\right)^{\frac{3}{2}}\right)=\sqrt{b\left(\frac{a+A}{2}\right)}
$$

In particular the mean iteration [4]

$$
A \otimes B(1, x)=1 / 2 F_{1}\left(\frac{1}{3}, \frac{2}{3} ; 1 ; 1-x^{3}\right) \text {. }
$$

\section{REFERENCES}

1. G. E. Andrews, The theory of partitions, Encyclopedia of Math. and Appl., Vol. 2 (G.-C. Rota, ed.), Addison-Wesley, Reading, Mass., 1976. (Reissued: Cambridge Univ. Press, London and New York, 1985.)

2. R. Bellman, A brief introduction to theta functions, Holt, Rinehart and Winston, New York, 1961.

3. B.C. Berndt, Ramanujan's notebooks, Part III, Springer-Verlag, New York, 1991.

4. J. M. Borwein and P. B. Borwein, Pi and the AGM - A study in analytic number theory and computational complexity, Wiley, New York, 1987.

5. $ـ$ A cubic counterpart of Jacobi's identity and the AGM, Trans. Amer. Math. Soc. 323 (1991), 691-701.

6. _ A remarkable cubic iteration, Computational Methods and Function Theory, Lecture Notes in Math., vol. 1435, Springer-Verlag, New York, 1990.

7. J. A. Ewell, On the enumerator for sums of three squares, Fibonacci Quart. 24 (1986), 151-153.

8. N. J. Fine, Basic hypergeometric series and applications, Math. Surveys and Monographs, No. 27, Amer. Math. Soc., Providence, R. I., 1988.

9. O. Kolberg, Note on the Eisenstein series of $\Gamma_{0}(p)$, Universitet i Bergen Arbok, Naturvitenskapelig rekke, Nr. 15, 1959.

10. L. W. Kolitsch, $A$ congruence for generalized Frobenius partitions with 3 colors modulo powers of 3, Analytic Number Theory, Proc. Conf. in honor of Paul T. Bateman, (B. C. Berndt et al. ed.), Birkhäuser, Boston, Mass., 1990.

11. L. Lorenz, Bidrag til tallenes theori, Tidsskrift for Mathematik (3) 1 (1871), 97-114.

12. S. Ramanujan, Notebooks, 2 vols., Tata Institute of Fundamental Research, Bombay, 1957.

13. _ The lost notebook and other unpublished papers, with an introduction by G. E. Andrews, Narosa Publishing House, New Delhi, 1988, (North American and European distribution: Springer-Verlag).

14. B. Schoeneberg, Elliptic modular functions, Springer-Verlag, New York, 1974.

Mathematics, Statistics and Computing Science Department, Dalhousie University, Halifax, N.S. B3H 3J5, CanAda

Current address, J. M. Borwein and P. B. Borwein: Centre for Experimental \& Constructive Mathematics, Simon Fraser University, Burnaby, British Columbia, Canada V5A 1S6

E-mail address: jborwein@cecm.sfu.ca, pborwein@cecm.sfu.ca

Current address, F. G. Garvan: Department of Mathematics, University of Florida, Gainesville, Florida 32611

E-mail address: frank@math.ufl.edu 